\title{
Análisis documental sobre los riesgos y las posibilidades de internet para los menores. Pautas educativas dirigidas a familias
}

\section{Bibliographical analysis on the risks and internet possibilities for minors. Educational guidelines addressed to parents}

\author{
María Ángeles Hernández Prados \\ Universidad de Murcia (España) \\ mangeles@um.es \\ Patricia López Vicent \\ Universidad de Murcia (España) \\ patlopez@um.es \\ Gonzalo Gamboa Gil de Sola \\ Broadwater School (Reino Unido) \\ ggildesola@gmail.com
}

Recibido: 01/06/2020

Aceptado: $22 / 03 / 2021$

Publicado: 01/06/2021

\section{RESUMEN}

Este trabajo presenta un análisis de la literatura científica sobre las consecuencias que tiene el uso que hacen los menores de las Tecnologías de la Información y la Comunicación (TIC). Para ello, se ha realizado una investigación de tipo teórica-bibliográfica, recurriendo a bases de datos de índole nacional e internacional, para obtener un total de 36 documentos que responden a los descriptores "familia" e "Internet" y a los criterios de inclusión-exclusión empleados, a partir de los cuales se han recogido riesgos y posibilidades para los menores de la red. Los datos han sido extraídos mediante una rejilla de vaciado de contenido, a partir de los cuales se concluye que, en los artículos analizados, los riesgos más generalizados son es el acceso al contenido nocivo, el ciberacoso y la adicción a la red, mientras que las posibilidades más destacadas que ofrece Internet son la creación de una identidad digital, el uso educativo de la red y las relaciones sociales que se pueden establecer a través de la misma. Finalmente, se lleva a cabo una propuesta de pautas educativas dirigidas a familias que permitan poner en práctica las posibilidades educativas y mitigar los principales riesgos de Internet que exponen las publicaciones estudiadas.

\section{PALABRAS CLAVE}

Menores; Internet; TIC; educación; familia.

\section{ABSTRACT}

This paper presents an analysis of the scientific literature on the consequences of children's use of Information and Communication Technologies (ICT). For this purpose, a theoretical-bibliographic research has been carried out, using databases of national and international nature, to obtain a total of 36 documents that respond to the descriptors "family" and "Internet" and to the inclusion criteria- employee exclusion, from which risks and possibilities for minors in the network have been collected. The data has been extracted through a content dump grid, from which it is concluded that, in the articles analyzed, the most widespread risks are access to harmful content, cyberbullying and network addiction. However, the most important possibilities offered by the Internet are the creation of a digital identity, the educational use of the network 
and the social relationships that can be established through it. Finally, a proposal of educational guidelines aimed at families that allow implementing the educational possibilities and mitigate the main Internet risks that expose the publications studied is carried out.

\section{KEYWORDS}

Children; Internet; ICT; education; family.

\section{CITA RECOMENDADA}

Hernández, M.A., López, P. \& Gamboa, G. (2021). Análisis documental sobre los riesgos y las posibilidades de internet para los menores. Pautas educativas dirigidas a familias. Revista Interuniversitaria de Investigación en Tecnología Educativa, 10, 9-22. https://doi.org/10.6018/riite.430341

\section{Principales aportaciones del artículo y futuras líneas de investigación:}

- El artículo presenta un análisis profundo de la literatura científica sobre riesgos y posibilidades que ofrecen las redes a los menores teniendo en cuenta el amplio volumen de publicaciones revisadas.

- El artículo ofrece una serie de pautas educativas dirigidas a familias a partir de los resultados obtenidos en el estudio.

- Las futuras líneas de investigación podrían dirigirse al análisis de la literatura científica desde una perspectiva docente, concretamente, actitudes y percepción del profesorado en cuanto a riesgos y posibilidades para los menores de las redes telemáticas.

- Las futuras líneas de investigación pueden dirigirse igualmente a realizar un análisis de publicaciones a nivel internacional sobre los riesgos y posibilidades que ofrecen a los menores las redes telemáticas, estableciendo una comparación con las aportaciones realizadas a nivel nacional.

\section{INTRODUCCIÓN}

La tecnología ha sido siempre un factor cultural y social que ha acompañado al ser humano desde sus inicios, mostrándose como una herramienta que facilita diversas tareas. Por norma, los cambios tecnológicos a lo largo de la historia han sido leves y lentos, limitados principalmente por los medios de cada época, pero ha sido en las últimas tres décadas cuando quizás su crecimiento y evolución han resultado más notables.

Hoy en día las Tecnologías de la Información y la Comunicación (TIC) son las herramientas más utilizadas en la sociedad para establecer vínculos y relaciones. Éstas se han convertido en una parte indispensable de nuestra forma de vivir, asociadas a un estilo de socialización más dinámico, instantáneo y cambiante. Las relaciones ya no quedan limitadas por el espacio ni por el tiempo: cualquier persona con los medios tecnológicos precisos puede ponerse en contacto de manera inmediata con otras personas sin importar su localización geográfica.

Sin duda, la aparición de las TIC en el ámbito social ha cambiado la manera que ha tenido siempre el mundo de comunicarse, y esto conlleva el surgimiento de nuevas corrientes de pensamiento sobre cómo abordar su uso, así como su utilidad en el desempeño de tareas de la vida diaria. Un tema que ha cobrado gran importancia en estos últimos años es el uso que los menores (considerando en este grupo a jóvenes, a niños y niñas y a adolescentes) hacen de las TIC en muchos ámbitos de su vida (Livingstone \& Haddon, 2008; Ruiz et al., 2013), con una consecuente demanda de la necesidad de realizar un uso seguro, en especial de Internet (Sureda et al., 2010).

Su popularidad entre el colectivo de jóvenes, adolescentes y niños acrecienta la palpable preocupación que tiene la sociedad por las nuevas generaciones, las cuales han nacido inmersas en el mundo de las TIC y sobre las que, en ocasiones, se pone en duda que hagan un uso adecuado de las mismas. A pesar de los beneficios que reportan, un uso extralimitado y poco responsable que atente contra el desarrollo cotidiano saludable del menor, perjudicando su 
evolución personal, escolar y social, es calificado cuanto menos de problemático. Aunque no se trata de un fenómeno muy generalizado, sí deben contemplarse acciones de sensibilización y concienciación social que actúen de modo preventivo, ya que genera impactos negativos en el ámbito académico (distracciones en clase y propiciar la disminución del tiempo dedicado al estudio) y afectan negativamente a la dimensión socio-relacional de los menores, tanto en el entorno social y familiar como de ocio (Díaz-Vicario et al., 2019).

Según, García et al. (2014) el uso excesivo de Internet puede contribuir a desarrollar en los menores una elevada confianza hacia las TIC que se relaciona con la normalización de diversas fórmulas de ciberbullying y una alta exposición involuntaria a contenidos inapropiados online como la pornografía, violencia, la promoción del alcohol y drogas o patologías alimentarias. En consonancia, algunos expertos afirman que los riesgos que se mencionan frecuentemente en relación al uso de Internet por parte de los menores son el acceso a contenidos inapropiados violentos o pornográficos-, el contacto con desconocidos o un mal uso de los datos personales (Bringué \& Sádaba, 2011; Hasebrink et al., 2009).

La importancia de los riesgos en la sociedad postindustrial ha sido puesta de manifiesto por diversos autores, convirtiéndose en un elemento clave en el estudio de los problemas sociales derivados de la sociedad moderna (Bonoli, 2007; Ivanova \& Ivanov, 2020; Luhmann, 1996), pues los riesgos que hoy irrumpen no son producidos por la producción y distribución de bienes y valores, sino por la producción y distribución de los peligros (realmente existentes) y los miedos (subjetivamente existentes) que se autoreplican, lo que conlleva una fase de reproducción, distribución y consumo de riesgos (Kryvoshein, 2018). Por riesgo se entiende la "posibilidad de que acciones humanas $u$ otras circunstancias impliquen consecuencias que afecten a diferentes aspectos que son valorados por las personas", definición que originalmente pertenece a Kates, Hohene \& Kasperson (1985 citado en Renn \& Benighaus, 2013, p. 295), distinguiéndose este concepto del de daño. La evidencia de riesgo no es, en sí misma, la evidencia de un daño real (Hasebrink et al.,2009) y las comparativas estadísticas muestran una incidencia del daño declarado en Internet por los menores muy diferente en función de los distintos riesgos y niveles de uso a los que se enfrentan, así como reacciones diversificadas para responder a situaciones heterogéneas.

En este sentido, los riesgos en general y las posibles soluciones a los problemas derivados de su uso, han sido puestos de manifiesto en los trabajos de Caro \& Plaza (2016) cuando abordan la adicción en los menores, o en el estudio de Yilmaz et al. (2017) sobre la seguridad en Internet y la percepción de los menores al respecto.

Respecto a la exposición a determinados riesgos por parte de los menores, la familia es uno de los pilares fundamentales, ya que forma parte del desarrollo del adolescente y participa activamente en su labor educativa. Según Coleman \& Hendry (2003), las familias se han preocupado tradicionalmente por el uso en la adolescencia de drogas, alcohol y tabaco, la delincuencia y ciertas conductas sexuales, elementos inapropiados en personas en proceso de crecimiento que en ocasiones buscan generar una imagen de sí mismos de madurez 0 , a veces, como medio para lograr la aceptación e inclusión en el grupo de iguales. A estas preocupaciones tradicionales, que emanan del deber y sentimiento de responsabilidad parental, se suman aquellas que se desprende de la seguridad de los menores en la red y su exposición ante los riesgos asociados a los usos de la misma.

Sin embargo, el relativo desconocimiento -aunque vaya decreciendo- que todavía tienen muchos padres y madres sobre Internet (Dowdell, 2012) y las limitaciones de los centros escolares, tanto en infraestructuras como en personal preparado al respecto, hacen que aún nos encontremos lejos de unos niveles deseables de alfabetización mediática suficientemente crítica y ajustada a una situación de consumo en constante evolución. De ahí que los menores suelan recurrir al aprendizaje entre iguales, dentro y fuera de la familia, que constituye un factor elemental para entender el uso que los más jóvenes hacen de Internet y al que apenas se le ha prestado atención (Livingstone \& Haddon, 2008) y que, sin duda, está relacionado con la percepción de los riesgos que tienen los propios menores.

En cualquier caso, hay un consenso creciente en relación a la necesidad de formar a los menores con el fin de que sean capaces de afrontar los riesgos de Internet por sí mismos y, del mismo modo, un consenso sobre la consideración de que la puesta en práctica de medidas 
excesivamente restrictivas orientadas a limitar los riesgos puede repercutir negativamente en el disfrute de oportunidades (Garmendia et al., 2012). Asimismo, hay que prestar atención a las capacidades de los menores para superar situaciones potencialmente peligrosas (Livingstone \& Helsper, 2008). La responsabilidad de esta formación no recae exclusivamente en los centros educativos de forma aislada, sino que requiere actuaciones colaborativas desarrolladas entre familias y escuela, para que estas iniciativas adquieran coherencia y mayores garantías de éxito.

\section{MÉTODO}

\subsection{OBJETIVOS}

El estudio realizado se lleva a cabo con el propósito general de analizar el estado de la cuestión sobre el uso de las redes telemáticas por parte del colectivo de menores que permita proponer medidas parentales que puedan contribuir a mejorarlo. Para ello, se formulan los siguientes objetivos específicos:

1. Analizar las posibilidades que ofrecen las redes telemáticas a los menores que se ponen de manifiesto en las publicaciones estudiadas.

2. Conocer los riesgos que habitualmente se asocian a la seguridad de los menores en la red descritos en las fuentes documentales analizadas.

3. Plantear pautas educativas dirigidas a familias que permitan, por un lado, poner en práctica las posibilidades educativas que ofrecen las TIC mencionadas en las referencias analizadas y por otro, mitigar los principales riesgos que exponen las publicaciones estudiadas con el fin de potenciar la seguridad de los menores en la red.

\subsection{SELECCIÓN DE UNIDADES DOCUMENTALES DE ANÁLISIS}

Para la búsqueda de la documentación necesaria se ha acudido a bases de datos de carácter científico, relacionadas principalmente con el ámbito de las Ciencias Sociales, tanto de carácter nacional (Dialnet, ISOC o Catálogo ALBA) y de carácter internacional (SCOPUS y ERIC). En el caso de las bases de datos españolas, los descriptores empleados son "familia" e "Internet", y para las bases de datos internacionales, "Internet", "security", "safety" y "parents", pues la generalidad de los términos "family" o "teen" dificultaba considerablemente la selección, generando un volumen amplio de artículos alejados del objeto del estudio. De hecho, con el descriptor "family" en el buscador de SCOPUS aparecían 16.000 resultados, de los cuales no todos hacían referencia a los adolescentes y al uso de la tecnología. Lo mismo sucedía con los descriptores "teen" o "teenager".

Los resultados obtenidos han sido recogidos en la Tabla 1, en la que también se muestran los criterios de inclusión-exclusión, que además de la repetición, contemplan el año de la producción, descartando todos aquellos anteriores al 2000 y aquellos artículos a los que no se pudo tener acceso al texto completo. Concretamente, el ajuste a la temática, año de publicación, repetición y acceso al documento completo ha permitido delimitar el número de documentos a 36 artículos.

Los documentos seleccionados abarcan una temporalidad de 2000 a 2018, siendo 2016 el que concetra mayor producción (22,22 \%), y una menor producción en los años más alejados en el tiempo, lo que denota que es un tema de actualidad reciente. En lo que respecta al tipo de estudios, las unidades documentales son variadas: revisión bibliográfica $(8,57 \%)$, el resto son de tipo empírico, de las cuales predomina las investigaciones cualitativas $(43,7 \%)$, seguidas de las cuantitativas $(31,2 \%)$ y de las mixtas $(25 \%)$. Centrándonos en la autoría, los artículos seleccionados son mayoritariamente elaborados por mujeres $(63,38 \%)$ y de forma colaborativa $(57,14 \%)$, predominando la realización de dos o tres autores. Finalmente, el 54,3\% son nacionales. 
Tabla 1. Frecuencia de documentos que responden al descriptor "familia e internet" por base de datos

\begin{tabular}{ccccccc}
\hline $\begin{array}{c}\text { Base de } \\
\text { datos }\end{array}$ & Resultados & Temática & Repite & Año & $\begin{array}{c}\text { Documento } \\
\text { Completo }\end{array}$ & $\begin{array}{c}\text { Número } \\
\text { "n" }\end{array}$ \\
\hline ISOC & 55 & 18 & 0 & 18 & 16 & 16 \\
Dialnet & 496 & 21 & 4 & 17 & 11 & 11 \\
$\begin{array}{c}\text { Catálogo } \\
\text { ALBA }\end{array}$ & 30 & 1 & 1 & 1 & 1 & 0 \\
ERIC & 61 & 14 & 0 & 14 & 8 & 8 \\
APA & 84 & 1 & 0 & 1 & 1 & 1 \\
$\begin{array}{c}\text { SCOPUS } \\
\text { (Elsevier) }\end{array}$ & 68 & 1 & 0 & 1 & 0 & 0 \\
& 1756 & 56 & 5 & 52 & 36 & 36 \\
\hline
\end{tabular}

\subsection{INSTRUMENTO}

Para el análisis de la información se ha utilizado una parrilla de elaboración ad hoc, validada por un grupo de expertos del área de metodología, de Tecnología Educativa y de teoría de la educación especializados en familia, compuesto por 11 indicadores que se distribuyen en dos dimensiones, riesgos de la red y posibilidades (Tabla 2). Para el proceso de validación se administró un cuestionario mixto a un total de ocho expertos, en su mayoría de género femenino $(75 \%)$ de diferentes áreas departamentales de la Universidad de Murcia (Teoría de la Educación, Diagnostico y Métodos de investigación, y Didáctica y Organización Escolar) y con más de 10 años de experiencia docente. Los criterios que se contemplan en el instrumento de validación son: claridad, adecuación y pertinencia de los objetivos, dimensiones y variables contempladas de forma cuantitativa en la parrilla, disponiendo de cuatro criterios de respuesta que oscilan de nada a mucho, y de un apartado específico para recoger de forma cualitativa propuestas de mejora.

Tabla 2. Dimensiones y variables

\begin{tabular}{lll}
\hline \multicolumn{1}{c}{ Dimensiones } & \multicolumn{1}{c}{ Variables } \\
\hline Riesgos de los menores en la red & - Acoso, Bullying o Ciberbulling \\
& $\bullet$ Pornografía, sexting o exposición sexual \\
& $:$ Uso irresponsable \\
& - Adicción a Internet \\
Posibilidades que ofrece Internet & - Búsqueda de información \\
& $:$ Relaciones sociales \\
& $:$ Creación de una adecuada identidad digital \\
& - Recurso para el aprendizaje \\
&
\end{tabular}

\subsection{PROCEDIMIENTO Y ANÁLISIS DE DATOS}

Para la obtención de los datos se ha contemplado, como medida principal, el número de veces que se hace referencia a los riesgos y posibilidades de Internet en la redacción de cada uno de los textos objeto de análisis. Esto incluye la relación recíproca entre datos dentro de cada documento o las posibles consecuencias derivadas, obtenidas como resultado de la propia investigación. Es por esto que la magnitud estadística que cobra mayor relevancia es la 
Frecuencia, como medida para determinar cuál es el riesgo o la posibilidad que ofrece la red que más se menciona en la documentación revisada.

Por otro lado, en lo estudios documentales en los que se realiza un análisis de la frecuencia en la que aparecen unas determinadas palabras se recurre al cálculo del peso semántico para poder favorecer la comparativa entre las unidades muéstrales del estudio. Siguiendo trabajos como el de Vera et al. (2005), se ha calculado el peso semántico (o importancia) de cada indicador en función de las repeticiones que se presenten de un parámetro en cada artículo revisado individualmente para, posteriormente, establecer un porcentaje con las frecuencias relativas dentro de cada publicación (es decir, cada artículo contará con un total de $100 \%$, teniendo en cuenta todos los parámetros que aparecen en él). El sumatorio del peso de los porcentajes ha permitido conocer la cantidad de artículos en los que tanto riesgos como posibilidades de Internet han sido mencionados. Si se divide este peso entre el número de artículos obtenidos, se obtendrá el porcentaje de importancia de cada uno de los parámetros a nivel general. Esto ha permitido analizar la información y poder así confeccionar orientaciones que se ajusten a estos datos.

\section{RESULTADOS}

En la Tabla 3 se destaca el sumatorio de los porcentajes teniendo en cuenta las frecuencias. Si dividimos este valor $(3200,00)$ entre 100 (relativo a $100 \%$ ), se obtiene la cifra de 32 , que representa el número de artículos en los que al menos uno de estos riesgos se ha mencionado o tratado. Por lo tanto, es necesario comentar que de entre los 36 artículos revisados, en 32 de ellos se ha podido encontrar una mención real a los riesgos de Internet, y que los resultados obtenidos se exponen en función de este muestreo.

Tabla 3. Información relativa a los riesgos de Internet (parámetros negativos)

\begin{tabular}{|c|c|c|c|c|}
\hline Núm & Parámetro & Frecuencia & $\begin{array}{c}\Sigma \\
\begin{array}{c}\text { Peso/documento } \\
(\%)\end{array}\end{array}$ & Peso (\%) \\
\hline 1 & $\begin{array}{l}\text { Acoso, Bullying o } \\
\text { Ciberbulling }\end{array}$ & 14 & 368,86 & 11,54 \\
\hline 2 & $\begin{array}{c}\text { Pornografía, } \\
\text { sexting o } \\
\text { exposición sexual }\end{array}$ & 21 & 533,22 & 16,67 \\
\hline 3 & Falsa identidad & 21 & 554,51 & 17,34 \\
\hline 4 & Uso irresponsable & 20 & 581,76 & 18,18 \\
\hline 5 & $\begin{array}{l}\text { Adicción a } \\
\text { Internet }\end{array}$ & 12 & 299,30 & 9,36 \\
\hline \multirow[t]{2}{*}{6} & Contenido nocivo & 24 & 829,02 & 25,91 \\
\hline & & & 3200,00 & 100,00 \\
\hline
\end{tabular}

Tal y como puede apreciarse en estos datos (Tabla 3), el riesgo que más se menciona en todos los artículos es el de "Contenido nocivo", siendo su importancia o peso sobre el recuento total de casi un $26 \%$, seguido de otros riesgos como el "Uso irresponsable", la "Falsa Identidad" o la "Pornografía, sexting o exposición sexual" con porcentajes de un $18,18 \%$, un $17,34 \%$ y un 16,67 \% respectivamente. En contraposición, el "Ciberbullying" o la "Adicción a Internet" han obtenido valores más bajos de presencia en los artículos (11,54\% y 9,36\% respectivamente). Por tanto, los riesgos que más destacan son los riesgos relacionados con el desarrollo del 
adolescente, principalmente en el ámbito educativo, ya que tanto el acceso a contenidos inapropiados como el uso irresponsable de las TIC son asuntos relacionados directamente con los procesos educacionales de los menores y afectan directamente a las labores educativas de los padres en la sociedad actual.

Por otra parte, respecto a las posibilidades que Internet brinda a los menores, se ha recabado información relativa a la "Búsqueda de información", "Relaciones sociales", "Creación de una adecuada identidad digital", "Recurso para el aprendizaje" y, por último, la "Transmisión de valores", obteniendo referencias a estos contenidos en 31 de los 36 artículos que componen la muestra de análisis de este estudio.

Tabla 4. Información relativa a las posibilidades que ofrece Internet (parámetros positivos)

\begin{tabular}{ccccc}
\hline Núm & Parámetro & Frecuencia & $\begin{array}{c}\Sigma \text { Peso/documento } \\
(\%)\end{array}$ & Peso (\%) \\
\hline $\mathbf{1}$ & $\begin{array}{c}\text { Búsqueda de } \\
\text { información }\end{array}$ & 17 & 502,71 & 16,22 \\
$\mathbf{2}$ & $\begin{array}{c}\text { Relaciones } \\
\text { sociales } \\
\text { Creación de una } \\
\text { adecuada } \\
\text { identidad digital }\end{array}$ & 24 & 625,40 & 20,17 \\
$\mathbf{3}$ & $\begin{array}{c}\text { Recurso para el } \\
\text { aprendizaje }\end{array}$ & 17 & 1169,73 & 37,73 \\
$\mathbf{4}$ & $\begin{array}{c}\text { Transmisión de } \\
\text { valores }\end{array}$ & 6 & 653,89 & 21,09 \\
& & 148,27 & 4,79 \\
\hline
\end{tabular}

En la Tabla 4 puede observarse que de entre las posibilidades más notorias que puede ofrecer Internet, la "Creación de una adecuada identidad digital" $(37,73 \%)$ es, de todas las posibilidades, la que más veces aparece y la que más peso tiene en el recuento general de los datos de los documentos, sobrepasando un tercio del recuento total. Muy próximos a este parámetro aparece Internet como "Recurso para el aprendizaje" $(21,09 \%)$ o el uso de las TIC para las "Relaciones sociales" (20,17\%). Cabe destacar también, el peso que tiene la "Búsqueda de información" (16,22 \%) como una de las posibilidades que estas ofrecen.

Sin embargo, dentro de los parámetros con menos peso o relevancia de todas las posibilidades que ofrece Internet se encuentra el referido a la "Transmisión de valores" a través de las TIC (4,79\%), ya que únicamente fue contemplado en 6 de los 36 artículos.

\section{DISCUSIÓN Y CONCLUSIONES}

\subsection{DISCUSIÓN}

Gracias a la información obtenida se han podido determinar las consecuencias más frecuentes derivadas del uso que hacen los menores de Internet, manifestadas en forma de riesgos y posibilidades. Respecto a los riesgos, cabe señalar que los resultados obtenidos se asemejan en gran medida a los que se plantearon en la encuesta EU Kids Online a menores de entre 9 y 16 años y a sus padres y madres realizada en 2011, donde la clasificación es similar y donde se concluye que la naturaleza de los problemas no siempre puede determinarse de manera tan clara (Garmendia et al., 2011).

Uno de los riesgos más frecuentes a los que están expuestos los menores en la red es el acceso a contenido inadecuado, que generalmente está destinado a adultos, por caracterizarse como contenido de tipo violento, racista o sexista (Albero, 2002; Andréu, 2003; Berrocal, 2013). 
Este aspecto se ha agudizado considerablemente en los últimos años con el aumento y fácil exposición a contenido de alta actividad sexual y pornográfica (Ruiz-Corbella \& De-Juanas, 2013; Mercado et al., 2016), el cual también se presenta como uno de los riesgos que se contempla en los artículos analizados en el estudio (16,67\%).

Otro de los riesgos que entraña la red puesto de manifiesto en la producción científica es el emergente y cada vez más extendido problema del acoso en red o ciberbullying. Se trata de un fenómeno relativamente reciente, cuyo volumen de trabajos centrados exclusivamente en analizar el ciberbullying se ha incrementado considerablemente en la última década. Según Ortega et al. (2016), el ciberacoso guarda relación con una serie de variables familiares (más conflictos, menos cohesión familiar y menos expresividad) y escolares (deterioro de la autoestima académica, peor ajuste escolar, bajo sentimiento de afiliación con sus iguales, falta de confianza en el profesorado, etc.), situando la prevalencia de dicho ciberacoso en el 27,4\%. Por su parte, Levent \& Taçgin (2017) reconocen que el ciberbullying puede derivar en una gran diversidad de efectos que dañan la salud psicológica de las víctimas y en relación al cual el profesorado desempeña un papel fundamental que se basa principalmente en la prevención del mismo. Sin embargo, y a pesar de los efectos perversos del ciberacoso, este se encuentra moderadamente contemplado en los trabajos globales de seguridad de los menores en la red, con un peso relativamente medio-bajo en comparación con el resto (11,54\%).

Por otra parte, según los resultados de esta investigación, la adicción se muestra como un problema menor. Sin embargo, la Estrategia Nacional sobre Adicciones 2017-2024 (Ministerio de Sanidad, Consumo y Bienestar Social del Gobierno de España, 2018) hace alusión a la adicción sin sustancia, refiriéndose a "los juegos de apuesta online, videojuegos y otras adicciones a través de las Nuevas Tecnologías" (p. 27), algo que pone de manifiesto la creciente preocupación social que existe sobre este tipo de conductas derivadas de otro riesgo manifestado en la literatura científica analizada como es el uso irresponsable que se hace de Internet. Además, dicho informe indica que el perfil de los jugadores online es cada vez más joven y con más nivel de estudios, los cuales están desarrollando una verdadera adicción al juego online y a los videojuegos. De igual modo, se reconoce que la adicción guarda relación con ciertas rutinas nocivas, como el frecuente uso de la pornografía, a la vez que aumenta la probabilidad de asumir otros riesgos (Schoenmakers \& Van Rooij, 2012), como la falsa identidad en la red, tal y como se pone de manifiesto en el estudio realizado.

A pesar de los riesgos, Internet ofrece muchas posibilidades que suponen ventajas para los menores. En cualquier caso, en el análisis global, los porcentajes obtenidos sobre las posibilidades que ofrecen las redes telemáticas han sido menores que los obtenidos en relación a los riesgos.

No obstante, como ha quedado reflejado en los resultados, las medidas adecuadas para focalizar la acción educativa de las familias y los docentes se podrían dirigir al uso educativo de las TIC, lo que coincide con lo expuesto por Sánchez et al. (2017) al afirmar que el factor educativo es el más influyente en el desarrollo de hábitos saludables en Internet, o con Tejedor y Pulido (2012) que sostienen que es el medio más socorrido y demandado por la sociedad. Así mismo, se constata que Internet es una excelente plataforma para la comunicación, centrado principalmente en labores educativas y aprendizaje, y que existe una conciencia responsable por parte de las familias con respecto a los usos de Internet y la voluntad de querer transmitir a sus hijos esa misma conducta (Andréu, 2003).

Por otra parte, los resultados obtenidos con respecto a la creación de una adecuada identidad digital, la búsqueda de información y las relaciones sociales a través de las TIC, son similares, pues se mencionan en gran cantidad de los documentos, pero su relevancia/peso es poca. Por último, en relación a la educación en valores, a pesar de que resultan esenciales para el ser humano y la convivencia social, los datos obtenidos en esta investigación evidencian que la presencia en los trabajos analizados es la más baja de todas. Pensar en las posibilidades de Internet para la transmisión de valores es algo novedoso, pero tal y como exponen HernándezPrados et al. (2015), Internet puede emplearse como medio para la transmisión de los mismos, aunque sea una faceta todavía a explorar.

En líneas generales, la sexualidad en la adolescencia, los contenidos nocivos, la irresponsabilidad de sus acciones o de los usos que hacen de las herramientas o dispositivos 
móviles, son temas de preocupación parental que se arrastran del pasado, pero cuya búsqueda de respuesta perdura en el presente. Junto a estas inquietudes emergen otros no menos importantes, patologías recientes como la adicción a Internet o problemas como el ciberbullying, los cuales requieren no solo un conocimiento del fenómeno sino también actuaciones dirigidas a la prevención, gestión y afrontamiento.

\subsection{CONCLUSIONES: PAUTAS EDUCATIVAS DIRIGIDAS A FAMILIAS}

A partir de los resultados obtenidos en este estudio, y tomando como punto de partida las fuentes documentales analizadas, se exponen, a continuación, una serie de pautas educativas dirigidas a las familias que podrían, por un lado, mitigar los riesgos más frecuentes a los que están expuestos los menores con el uso de la red y por otro, potenciar las posibilidades que esta ofrece. Dado que de forma global, podemos concluir que los aspectos relacionados con los riesgos de Internet cuentan con una mayor presencia en los artículos que los aspectos relativos a las posibilidades de las TIC, de modo que predomina una visión negativa frente a la positiva, y tomando en consideración los efectos perjudiciales de la red, se ha estimado adecuado enfatizar más las medidas educativas relacionadas con los riesgos que las relativas a potenciar las posibilidades que ofrece Internet a los menores.

Las recomendaciones respecto a los riesgos se diferencian en función de su tipología, de ahí que tengan que ser expuestos individualmente, aunque en ciertos casos estas pautas puedan ser eficaces para prevenir diversos riesgos a la misma vez. En lo que respecta al acceso a contenido nocivo y a pornografía, amparado por el derecho a la libertad de expresión, los menores no siempre son capaces de distinguir el riesgo asociado al mismo. Existe la necesidad de proteger jurídicamente a los menores de los contenidos ilícitos e inapropiados para su edad (Reid, 2005), y las medidas que tradicionalmente se han recomendado a las familias para prevenir este tipo de acceso de los menores a contenido nocivo (moralmente reprochable) 0 ilegal se han centrado en la regulación del contenido de Internet a través de sistemas de clasificación y herramientas de filtrado.

En este sentido, en 1998 Akdeniz expuso la necesidad de hacer público y abierto el debate sobre la regulación del contenido nocivo en Internet para que no quedara en manos exclusivamente de los gobiernos y ciertas empresas privadas, pero la regulación mediante los sistemas de filtrado es poco objetiva, a veces resulta ruda y tiende a bloquear demasiados sitios web. Además, en ocasiones los menores logran desactivar dichas herramientas. Por todo ello, se considera que este tipo de medidas nunca deben suplantar la labor educativa de las familias, dirigida a promover el acompañamiento como sinónimo de estar presente, de habituar y naturalizar el diálogo paternofilial sobre las TIC, pero también dirigida a mostrar interés por los usos que los menores hacen de Internet, así como por los gustos y aficiones en la red, para poder así mediar de forma efectiva (Giones-Valls \& Serrat-Brustenga, 2010). Además, esta labor educativa de las familias se debe dirigir también a reflexionar sobre los tipos de contenido nocivo que podemos encontrar y recibir formación específica que favorezca, como señalan Marín y García (2003), la cooperación familia-docente para fomentar en el colectivo adolescente el desarrollo de un pensamiento crítico ante cualquier tipo de información presente en la red.

Por otra parte, la normalización de ciertos comportamientos ilegales en la red es bastante común entre los menores. Así pues, la descarga gratuita de juegos, música, series o películas es además de una práctica habitual en la e-generación, una acción ilegal (Livingstone \& Haddon, 2008), por lo que, según Barrio (2017), la Ley de Propiedad Intelectual (aprobado por Real Decreto Legislativo 1/1996, de 12 de abril) resulta insuficiente para frenarla. En base a lo expuesto concluimos que, los menores son grandes conocedores del uso y dominio de herramientas que posibilitan la consecución de sus propios fines e intereses, pero a la vez, son desconocedores de lo legal e ilegal, de lo tolerable e intolerable, y trasgreden con facilidad los límites, haciendo un uso irresponsable de la red

El desamparo de los menores ante ciertos riesgos en la red se visibiliza significativamente en el caso del ciberacoso, en el que el anonimato, la facilidad y diversidad de formas de ridiculizar y atentar contra otra persona y la impunidad del agresor dificultan una respuesta urgente correctiva del mismo (Hernández-Prados \& Solano, 2007). Para Garaigordobil (2011), los programas de intervención psicológica sobre bullying y ciberbullying en contextos educativos y 
la implementación de propuestas didácticas para la familia y la comunidad educativa entre los que cita a diversas guías como Kit Stop Ciberbullying, e-Legales, INTECO, etc. son algunas de las medidas que actualmente se están desarrollando.

Del mismo modo, el énfasis en la educación para la ciudadanía, transmitiendo la vivencia del respeto y la empatía, el desarrollo de la resiliencia familiar, así como la promoción de la escucha activa por parte de los adultos hacia los menores, son aspectos que favorecen por un lado la prevención de comportamientos violentos, y por otro, la identificación temprana de los mismos en el caso de las víctimas. La identidad ciudadana exige la inclusión de una adecuada identidad digital que ha de ser promovida desde diversos contextos. En esta misma dirección, Ruiz-Corbella \& De-Juanas (2013) exponen la necesidad de formar la identidad del menor en la red, enseñando a los menores a utilizar de manera responsable las TIC, es decir, aprender a gestionar el correo electrónico, mostrar cómo desarrollar una identidad profesional en las redes, etc. y recomienda trabajar primero sobre la identidad offline, es decir, sobre la identidad propia fuera de las redes.

En ocasiones, los menores desconocen la trascendencia que tienen sus acciones en la red, por ello, educar en la prudencia, a ser reflexivos y a tomar decisiones sopesando las consecuencias para reconocer situaciones de ciberacoso y saber cómo pedir ayuda (a la familia, a docentes, a la policía y a otros servicios) y cómo denunciarlas es fundamental. Al respecto, son varias las web de fácil acceso que permiten la denuncia de estos casos de acoso. No obstante, se considera esencial inculcar el sentido de la privacidad, esencialmente en las redes sociales y fomentar la seguridad de las acciones de los menores en Internet (Labrador et al., 2015).

La adicción es otro de los riesgos contemplados que, según Hernández-Prados et al. (2008), perjudica a los menores porque desajusta el ritmo de sus actividades diarias, les resta tiempo para dormir, asearse, hacer deporte o impide mantener una dieta equilibrada, pero también perjudica sus estudios o sus relaciones personales que es lo que más valoran ellos en estas edades, y lo más llamativo es que los propios menores son conocedores de estos efectos perversos, pero no saben cómo frenarlos. Esto efectos no deben ser tratados de manera individual, pues las patologías o trastornos normalmente son ocasionados por varios factores, entre otros, por una mala mediación o por una mala gestión de los recursos de Internet (Caro \& Plaza, 2016).

En un intento de reducir el tiempo que los menores pasan conectados, las familias deberían establecer espacios temporales diarios sin TIC, ofreciéndoles alternativas de ocio saludables que les motiven, ampliando el tiempo progresivamente para evitar crear cuadros de ansiedad o brotes de agresividad como consecuencia de la frustración que les genera no poder usarlos. Potenciar el diálogo familiar, las salidas familiares, la práctica de actividades deportivas y la planificación de actividades, contabilizando el tiempo invertido en cada una de ellas, ya que permitirá al menor tomar conciencia de su situación ante las TIC. A modo de ejemplo, consideramos de utilidad la Guía para padres y educadores sobre el uso seguro de Internet, móviles y videojuegos elaborada por Labrador et al. (2015) en la que aportan una serie de recomendaciones orientadas a unas restricciones o dosificación de la tecnología en el hogar bajo una supervisión parental.

En relación a las posibilidades que puede ofrecer a los menores el uso de Internet, se han identificado en este análisis bibliográfico dos grandes dimensiones, la comunicativa y la educativa. Desde el punto de vista comunicativo, la integración de las TIC en la vida ciudadana cambió la naturaleza del diálogo pues "la interacción es en simultáneo, a través de múltiples pantallas, en tiempo real y sin necesidad de presencia física" (Morduchowicz, 2012, p.11), siendo este el principal atractivo de las relaciones sociales, las cuales han sido contempladas en este trabajo como una fuente de posibilidades. En este sentido, el aislamiento promovido por la red ha sido desmitificado por un estudio que demuestra que los menores que más suelen salir con sus amigos (al cine, a discotecas, a pasear, etc.) son las que más utilizan Internet (López \& Solano, 2011). La preferencia de las posibilidades comunicativas mediadas tecnológicamente, a pesar del carácter informal de la comunicación, la imposibilidad de dar afecto a través de la red o la pérdida del componente gestual y del tacto en la comunicación a través de Internet, se limita, la mayoría de veces, al grupo de iguales, desaprovechando la comunicación con familiares (Hernández-Prados et al., 2015). 
Desde el punto de vista educativo, nos centraremos en la necesidad de impulsar la transmisión de valores y la creación de una adecuada identidad digital. Además de los valores que tradicionalmente impulsa la red de solidaridad, la colaboración, el diálogo y la creatividad, también se deben impulsar otros valores que favorecen un uso responsable de las TIC como la sinceridad, la honestidad, la prudencia, la justicia, la sensatez, el autocontrol, el respeto, la disciplina como cumplimento de las normas, entre otros. Favorecer la responsabilidad digital implica educar desde la norma, propiciar el desarrollo de habilidades comunicativas, y democratizar, como señala Morduchowicz (2012), las decisiones que toman los menores, dotándoles de la confianza necesaria para que realicen elecciones que les lleven a un uso responsable de Internet y a la creación de una adecuada identidad digital.

El reto educativo de las familias es saber cómo promover que los menores superen el mero uso comunicativo de la red para pasar a hacer un uso también educativo de la misma. Así, es importante que estos menores aprendan a establecer criterios para la selección de información, conozcan los mecanismos de búsqueda, selección, gestión y almacenamiento de información para realizar tareas escolares en casa, pero también que conozcan las principales herramientas para la colaboración y comunicación de tipo educativo, pues a menudo, encontramos entre los menores muy desarrollado el uso de Internet con fines dirigidos al ocio, las relaciones sociales 0 el entretenimiento, pero poco potenciado el uso educativo de las redes telemáticas.

\section{REFERENCIAS BIBLIOGRÁFICAS}

Akdeniz, Y. (1998). Who watches the watchmen? Internet content rating systems and privatised censorship. The Australian Library Journal, 47(1), 28-42. https://doi.org/10.1080/00049670.1998.10755831

Albero, M. (2002). Adolescentes e Internet. Mitos y realidades de la sociedad de la información. Revista de Estudios de Comunicación, 7(3). https://bit.ly/2Rg1JWk

Andréu, J. (2003). Infancia socialización familiar y nuevas tecnologías de la comunicación. Revista Portuaria, 3, 243-261. https://bit.ly/3cYKrFH

Barrio, M. (2017). Derecho Público y Propiedad Intelectual: su protección en Internet. Editorial Reus.

Beck, U. (1998). La sociedad del riesgo: hacia una nueva modernidad. Paidós.

Berrocal, A. I. (2013). La protección de los derechos de los menores de edad en Internet. Revista crítica de derecho inmobiliario, 89(739), 3371-3422.

Bringué, X. \& Sádaba, C. (2011). Menores y redes sociales. Colección Foro Generaciones Interactivas/Fundación Telefónica.

Bonoli, G. (2007). Time matters: Postindustrialization, new social risks, and welfare state adaptation in advanced industrial democracies. Comparative political studies, 40(5), 495-520. https://doi.org/10.1177/0010414005285755

Caro, P. J. \& Plaza, J. (2016). Intervención educativa familiar y terapia sistémica en la adicción adolescente a Internet: Fundamentación teórica. Revista Española de Orientación y Psicopedagogía, 27(1). https://doi.org/10.5944/reop.vol.27.num.1.2016.17031

Coleman, J. C. \& Hendry, L. B. (2003). Psicología de la adolescencia. Morata.

Díaz-Vicario, A., Mercader J. C. \& Gairín Sallán, J. (2019). Uso problemático de las TIC en adolescentes. Revista Electrónica de Investigación Educativa, 21(1), 1-11. https://doi.org/10.24320/redie.2019.21.e07.1882

Dowdell, E. B. (2012). Use of the Internet by parents of middle school students: Internet rules, risky behaviours and online concerns. Journal of Psychiatric and Mental Health Nursing, 20(1), 9-16. https://doi.org/10.1111/j.1365-2850.2011.01815.x 
Garaigordobil, M. (2011). Prevalencia y consecuencias del cyberbullying: una revisión. International journal of psychology and psychological therapy, 11(2), 233-254. https://bit.ly/3mahsNU

García, B., López, M. C. \& García, A. (2014). Los riesgos de los adolescentes en Internet: los menores como actores y víctimas de los peligros de Internet. Revista Latina de Comunicación Social, 69, 462-485. https://bit.ly/3wAclzk

Garmendia, M., Garitaonandia, C., Martínez, G. \& Casado, M.Á. (2012). The effectiveness of parental mediation. In S. Livingstone, L. Haddon y A. Görzig, (Eds.), Children, Risk and Safety Online: Research and Policy Challenges in Comparative Perspective (pp. 231-244). The Policy Press. https://bit.ly/39PIFEW

Garmendia, M., Garitaonandia, C., Martínez, G. \& Casado, M. Á. (2011). Riesgos y seguridad en Internet: Los menores españoles en el contexto europeo. Servicio editorial de la Universidad del País Vasco. https://bit.ly/3s2zPcR

Giones-Valls, A., \& Serrat-Brustenga, M. (2010). La gestión de la identidad digital: una nueva habilidad informacional y digital. BiD: textos universitaris de biblioteconomia i documentació, (24). https://bit.ly/3wAduqC

Hasebrink, U., Livingstone, S., Haddon, L. \& Olafsson, K. (2009). Comparing children's online opportunities and risks across Europe. School of Economics. https://bit.ly/31TaHe3

Hernández-Prados, M. Á., López, P. \& Bautista, V. (2015). La percepción del alumnado de educación secundaria sobre la transmisión de valores a través de las TIC. Revista Interuniversitaria de Teoría de la Educación, 27(1), 169-185. https://doi.org/10.14201/teoredu2015271169185

Hernández-Prados, M.A. \& Solano, I.M. (2007). Ciberbullying, un problema de acoso escolar. Revista Iberoamericana de Educación a Distancia, 10(1), 17-36. https://doi.org/10.5944/ried.1.10.1011

Hernández-Prados, M.A., López, P. \& Solano, I.M. (2008). Los menores y el ordenador: Educar en hábitos saludables. Cuadernos de Pedagogía, 380, 34-37.

Ivanova, S. \& Ivanov, O. (2020). Education in the Era of the Fourth Industrial Revolution: Development Vector, Prospects and Challenges for Russia. Space and Culture, India, 7(5), 70-79. https://doi.org/10.20896/saci.v7i5.703

Kryvoshein, V. (2018). Social risks of postmodernity: essence and classification. Epistemological Studies in Philosophy, Social and Political Sciences, 1(1-2), 16-28. https://doi.org/10.15421/341803

Labrador, F., Requesens, A. \& Helguera, M. (2015). Guía para padres y educadores sobre el uso seguro de Internet, móviles y videojuegos. Fundación Gaudium y Obra Social Caja Madrid. https://bit.ly/31R3sU9

Levent, F. \& Taçgin, Z. (2017). Examining Cyberbullying Tendency and Multidimensional Perceived Social Support Status of Teacher Candidates. Turkish Online Journal of Educational Technology-TOJET, 16(1), 37-46. https://bit.ly/3dGc8lz

Livingstone, S. \& Haddon, L. (2008). Risky Experiences for Children Online: Charting European Research on Children and the Internet. Children \& Society, 22(4), 314-323. https://doi.org/10.1111/i.1099-0860.2008.00157.x

Livingstone, S. \& Helsper, E.J. (2008). Parental mediation of children's internet use. Journal of Broadcasting \& Electronic Media, 52(4), 581-599. https://doi.org/10.1080/08838150802437396 
López, P. \& Solano, I.M. (2011). Interacción social y comunicación entre jóvenes. En F. Martínez y I.M. Solano (Eds.), Comunicación y relaciones sociales de los jóvenes en la red (pp. 27-46). Marfil.

Luhmann, N. (1996). El concepto de riesgo. En J. Beriain (Ed.), Las consecuencias perversas de la modernidad (pp. 123-153). Anthropos.

Marín, V. \& García, M. D. (2003). La familia e Internet, ¿un juego a tres bandas? Comunicar: Revista Científica de Comunicación y Educación, 11(21), 123-126. https://doi.org/10.3916/C21-2003-18

Mercado, C., Pedraza, F. J. \& Isela, K. (2016). Sexting: su definición, factores de riesgo y consecuencias. Revista sobre la infancia y la adolescencia, 10, 1-18. https://doi.org/10.4995/reinad.2016.3934

Ministerio de Sanidad, Consumo y Bienestar Social del Gobierno de España (2018). Estrategia Nacional sobre adicciones 2017-2024. https://bit.ly/2Rev1oi

Morduchowicz, R. (2012). Los adolescentes y las redes sociales La construcción de la identidad juvenil en Internet. Fondo de Cultura Económica de Argentina.

Ortega, J., Buelga, S. \& Cava, M. J. (2016). Influencia del clima escolar y familiar en adolescentes, víctimas de ciberacoso. Comunicar, 24(46), 57-65. http://dx.doi.org/10.3916/C46-2016-06

Reid, A. S. (2005). The rise of third generation phones: The implications for child protection. Information \& Communications Technology Law, 14(2), 89-113. https://doi.org/10.1080/13600830500042616

Renn, O. \& Benighaus, C. (2013). Perception of technological risk: insights from research and lessons for risk communication and management. Journal of Risk Research, 16(3-4), 293-313. https://doi.org/10.1080/13600830500042616

Ruiz-Corbella, M. \& De-Juanas Oliva, A. (2013). Redes Sociales, identidad y adolescencia: nuevos retos educativos para la familia. Estudios Sobre Educación, 25, 95-113. https://bit.ly/3uuQfMP

Ruiz, J. A., Ortiz, M. Á. \& Porto, L. (2013). Aportaciones de las universidades españolas a la investigación sobre menores y medios de comunicación. Anàlisi: Quaderns de comunicació $i$ cultura, 48, 49-64. https://bit.ly/3mzKtql

Sánchez, M., Frutos, B. \& Vázquez, T. (2017). La influencia de los padres en la adquisición de habilidades críticas en Internet. Comunicar, 15(53), 103-111. https://doi.org/10.3916/C53$\underline{2017-10}$

Schoenmakers, T. \& Van Rooij, T. (2012). Internet addiction in the Netherlands a brief overview. BELSPO.

Sureda, J., Comas, R. \& Morey, M. (2010). Menores y acceso a Internet en el hogar: Las normas familiares. Comunicar, 17(34), 135-143. https://doi.org/10.3916/C34-2010-03-13

Tejedor, S. \& Pulido, C. M. (2012). Retos y riesgos del uso de Internet por parte de los menores. ¿Cómo empoderarlos?. Revista Comunicar, 20(39), 65-72. https://doi.org/10.3916/C39-2012$\underline{02-06}$

Vera, J., Pimentel, C. \& Batista, F. (2005). Redes semánticas: aspectos teóricos, técnicos, metodológicos y analíticos. Ra Ximhai, 1(3), 439-451.

Yılmaz, R., Karaoğlan Yılmaz, F.G., Öztürk, H.T. \& Karademir, T. (2017). Examining secondary school students' safe computer and internet usage awareness: An example from Bartın province. Pegem Eğitim Ve Öğretim Dergisi, 7(1), 83-114. https://doi.org/10.14527/pegegog.2017.004 


\section{INFORMACIÓN SOBRE LOS AUTORES}

\section{María Ángeles Hernández Prados}

Universidad de Murcia

Licenciada y doctora en Pedagogía y Profesora Titular de Universidad en el Departamento de Teoría e Historia de la Educación de la Universidad de Murcia. La producción científica se encuentra relacionada con las siguientes líneas de investigación: educación para la ciudadanía y mejora de la convivencia escolar (Formación del profesorado, escuelas de padres, etc.), educación en valores en el contexto familiar (resolución de conflictos, aprendizaje de normas, implicación parental en las cuestiones escolares, educar en y desde la responsabilidad, etc.) y perspectiva ético-moral de la Sociedad de la Información (la seguridad de los menores en la red, ciudadanía digital, cyberbullying, etc.).

\section{Patricia López Vicent}

Universidad de Murcia

Licenciada y doctora en Pedagogía y Profesora Contratada Doctora en el Departamento de Teoría e Historia de la Educación de la Universidad de Murcia. Cuenta con proyectos de investigación nacionales e internacionales y numerosas publicaciones relacionados con la aplicación de las TIC en contextos socio-educativos: redes de colaboración docente, tutoría electrónica en educación superior, Educación en valores, familiar y TIC, educación intercultural y TIC, la seguridad de los menores en la red, ciudadanía digital, cyberbullying, etc.

\section{Gonzalo Gamboa Gil de Sola}

Broadwater School

Graduado en Arquitectura Técnica por la Universidad Politécnica de Cartagena. Máster universitario en Formación del Profesorado. Actualmente es profesor en el centro de educación secundaria Broadwater Shool en Godalming, Inglaterra (Reino Unido).

\section{(c) (8)}

Los textos publicados en esta revista están sujetos a una licencia de Reconocimiento 4.0 España de Creative Commons. Puede copiarlos, distribuirlos, comunicarlos públicamente y hacer obras derivadas siempre que reconozca los créditos de las obras (autoría, nombre de la revista, institución editora) de la manera especificada por los autores o por la revista. La licencia completa se puede consultar en: Licencia Creative Commons Atribución-NoComercial-Compartir por igual 4.0 Internacional 